\title{
Thinking Outside the Box: Agile Business Models for CNOs
}

\author{
Leandro Loss and Servane Crave
}

\author{
Orange Labs R\&D \\ 905, rue Albert Einstein, 06921, Sophia Antipolis Cedex, France \\ \{leandro. loss, servane. crave\} @orange-ftgroup.com
}

\begin{abstract}
This paper introduces the idea of an agile Business Model for CNOs grounded on a new model of innovation based on the effects of globalization and of Knowledge Economy. The agile Business Model considers the resources that are spread out and available worldwide as well as the need for each customer to receive a unique customer experience. It aims at reinforcing in the context of the Knowledge Economy the different business models approaches developed so far. The paper also identifies the levers and the barriers of Agile Business Models Innovation in CNOs.
\end{abstract}

Keywords: Collaborative Networks, Agile Business Models, Customer Experience.

\section{Introduction}

Collaborative Networked Organizations (CNOs) (Camarinha-Matos and Afsarmanesh, 2005) are assessed as organizational forms with an appropriate compliance in a context of globalization and turbulent markets. This adaptability is due to their fast and reliable arrangement capacities with regards to the collaboration opportunities that occur or that they are able to detect.

One can observe that CNOs have been well described and documented thanks to real case studies (in Aeronautics, Automotive networks) and trends of research explored in European projects, such as ECOLEAD (theoretical and technological foundations for CNOs), ATHENA and INTEROP (interoperability issues and seamless business interactions across organizational boundaries). However, the CNO field of research is not yet complete; rather it still presents many issues to be further explored. One of these fields is related to the BM studies.

In Marketing, BMs have become in the last decade a fashionable term to describe the way an organization is going to address the market. In Organizational Sciences, it is presented as a conceptual tool composed of several interrelated building blocks which aim at defining the product/service, the revenue model, the value proposition, the distribution channel, that a single organization offers to one or several segments of customers (Osterwalder, 2004). It is of evidence that CNOs already make use of BMs when they are elaborating their strategy. However, proven BMs approaches like Chesbrough et al. (2002) and Osterwalder (2004) are rather static in the sense that 
they are designed for a single organization with a linear value chain, within a stable and predictable competitive landscape. Romero et al. (2006) attempted to cope with this issue through a multi perspective approach regarding BMs for CNOs focusing on two different features: the Value Proposition and the nature of the shareholders ${ }^{1} \&$ stakeholders $^{2}$. Romero et al. (2006) is a noticeable asset but it could be enhanced in order to support CNOs in a context of globalization and increased uncertainty.

The aforementioned BM approaches face difficulties to capture the necessary shift in the new ways of working of CNOs' stakeholders, mainly regarding innovation aspects. Consequently, an agile BM strategy is required for CNOs. This paper proposes to ground it on a new model of innovation which finds its roots in a context where resources are spread out worldwide and where each customer will receive a unique experience. Prahalad et al. (2008) have extensively developed the idea of $\mathrm{R}=\mathrm{G}$ (access to high-quality resources at low cost) and $\mathrm{N}=1$ (the focus is on the individual consumer). These concepts are mobilized in this paper in order to create an agile Business Model for CNO's. This research work also includes the identification of innovation levers and barriers when defining BMs for CNOs.

The paper is organized as follows: section 2 presents the CNOs approach as a sound alternative in markets dominated by globalization and turbulence. Section 3 presents a joint perspective of the BM approaches introduced by Osterwalder (2004), Romero et al. (2006), and of empirical tools used to support BMs elaboration. Section 4 presents the levers that lead to innovation in BMs as well as the related uncertainties. To conclude, section 5 brings the final remarks of this work and opens new research avenues.

\section{CNOs: An Alternative for Turbulent Markets}

Since mid-2007 the economic world has been facing the so called "credit crunch". This phenomenon started with the lack of trust by investors in the value of securitized mortgages ${ }^{3}$ in the USA. After that the crisis has deepened and affected the stock markets all around the world (with very few exceptions). Many financial institutions have collapsed or were nationalized being under federal regulations. Countries that have not been in recession (negative real economic growth) for many years are now also facing this problem. Both US Federal Reserve and the European Union countries have made efforts to minimize the problems caused by this unprecedented crisis.

On the one hand, recession is often synonymous of factories closing-down and employees' layoffs. On the other hand, as stated by Papadakis et al. (1999) and Stevenson and Jarillo (1990), periods of crisis can also be seen as moments for entrepreneurial growth, where organizations should be looking for new opportunities, rather than defending existing resources.

\footnotetext{
${ }^{1}$ A "shareholder" is an individual or company that legally owns one or more shares of stock in a joint stock company. A company's shareholders collectively own that company.

${ }^{2}$ A "stakeholder" is a person, group, organization, or system who affects or can be affected by an organization's actions.

${ }^{3}$ Securitized mortgages involve the pooling and repacking of cash flow producing financial assets into securities that are then sold to investors (Raynes et al., 2003).
} 
One option to better explore opportunities and react to turbulent markets, mainly for Small and Medium sized Enterprises (SMEs), is to work collaboratively in strategic alliances. Lendrum (1997) defines a strategic alliance as a formal relationship that rises up from two or more participants in order to pursue a set of agreed goals or to meet a critical business. Oliver (1990) reviewed the literature on inter-organizational relations and argues that the core idea is that organizations working together are stronger than when working only by themselves or isolated. According to Loss et al. (2008), strategic alliances are also viewed as a form of Collaborative Networked Organization (CNOs).

Camarinha-Matos and Afsarmanesh (2006) argue that CNOs provide access to new/wider markets and novel knowledge, where risks and resources are shared and where skills and capacities can be complementary orchestrated. Therefore entities being part of a CNO may focus on their core competencies while keeping high level of agility. New organizational forms also lead to innovation, and thus creation of new value by confrontation of ideas and practices, combination of resources and technologies, and creation of synergies (Camarinha-Matos and Afsaramanesh, 2006).

CNOs are per nature dynamic and adaptive organizational forms, but they also need to be continuously aware of the non-stable and unpredictable competitive landscape. With a view to better explore market opportunities and improve their competitiveness, CNOs Managers shall not anymore rely only on the traditional BMs. They shall, therefore, look for dynamic supporting tools that allow the elaboration of new and innovative approaches in order to conquest new markets. It means to better adapt themselves to changes.

\section{Current Business Models Approaches}

According to Chesbrough and Rosenbloom (2002) the key to release value from a service or a technology is a successful Business Model. It means that the business model perspective takes into consideration the ways in which services and/or technologies can be valuable. The functions of a business model, according to these authors, are:

- Identify a market segment. The users to whom the technology is useful and for what purpose;

- Articulate the value proposition. The value created for users by the offering based on the technology;

- Define the structure of the value chain within the firm required to create and distribute the offering;

- Estimate the cost structure and profit potential of producing the offering, given the value proposition and value chain structure chosen;

- Describe the position of the firm within the value network linking suppliers and customers, including identification of potential complements and competitors;

- Formulate the competitive strategy by which the innovating organization will gain and hold advantage over rivals.

As a result $\mathrm{BM}$ is seen as the linking element from technical inputs to economic outputs (Fig. 1). 

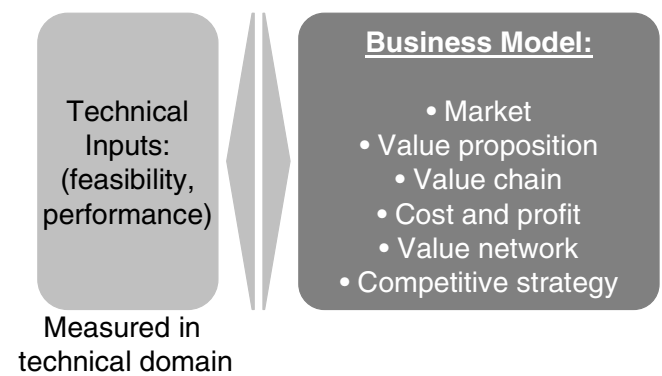

technical domain

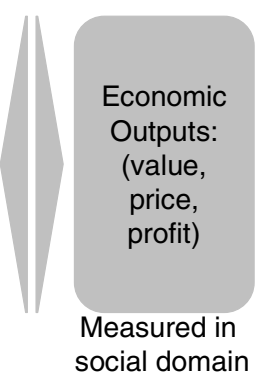

Fig. 1. Business Model Mediates Between the Technical and Economic Domains. Source: Chesbrough and Rosenbloom (2002).

It is understood that business models should embrace a wider perspective that also includes possible partnerships, customer requirements, revenue shares and other elements. Osterwalder (2004) defines Business Models as:

"... a conceptual tool that contains a set of elements and their relationships and allows a company's logic of earning money. It is a description of the value a company offers to one or several segments of customers and the architecture of the firm and its network of partners for creating marketing and delivering this value and relationship capital, in order to generate profitable and sustainable revenue streams".

Looking for a formal way to accurately describe the business model of a firm, Osterwalder (2004) identified four main pillars seen as the basis of a BM: product (what?), customer interface (who?), infrastructure management (how?), and financial aspects (how much?). These four areas were broken down in nine interrelated building blocks: value proposition, under the pillar product; target customer, distribution channel, and relationship, under the pillar customer interface; value configuration, capability, and partnership, under the pillar infrastructure management; and cost structure and revenue model, under the pillar financial aspects. Romero et al. (2006) extended Osterwalder's work to CNOs introducing the idea of multi-value proposition and configuration to match the nature of CNOs. For supporting development of new services in the ICT sector. Plantin et al., (2008), have developed an exhaustive check-list of all relevant actions when developing a Business model including: ecosystem analysis understanding the scope of the relevant field of economic activity; breakthrough anticipation - possible events that can change a value chain; competitive analysis - identifies the various competitors; pre-segmentation and segmentation - main strengths and weaknesses of the value perceived by the customer; business analysis - identification of the main values, key success factors, know-how, and critical resources; fields of innovation and value creation; market analysis - characterization of the target market in both qualitative and quantitative ways; value chain - understand the logic of a of activities performed; supply strategy - describe the services in order to make them intelligible; value proposition; and SWOT analysis. 
Fig. 2 shows a graphical representation that merges the model for BM introduced by Ostervalder (2004) and improved by Romero et al. (2006). Additionally, the empirical-based marketing approaches that support the elaboration of BMs (Plantin et al., 2008) are also included. This graphical representation is in accordance with Chesbrough and Rosenbloom (2002) proposition where the BM is placed as a bridging element that links technical inputs (infrastructure management) and economical outputs (customer interface and financial aspects).

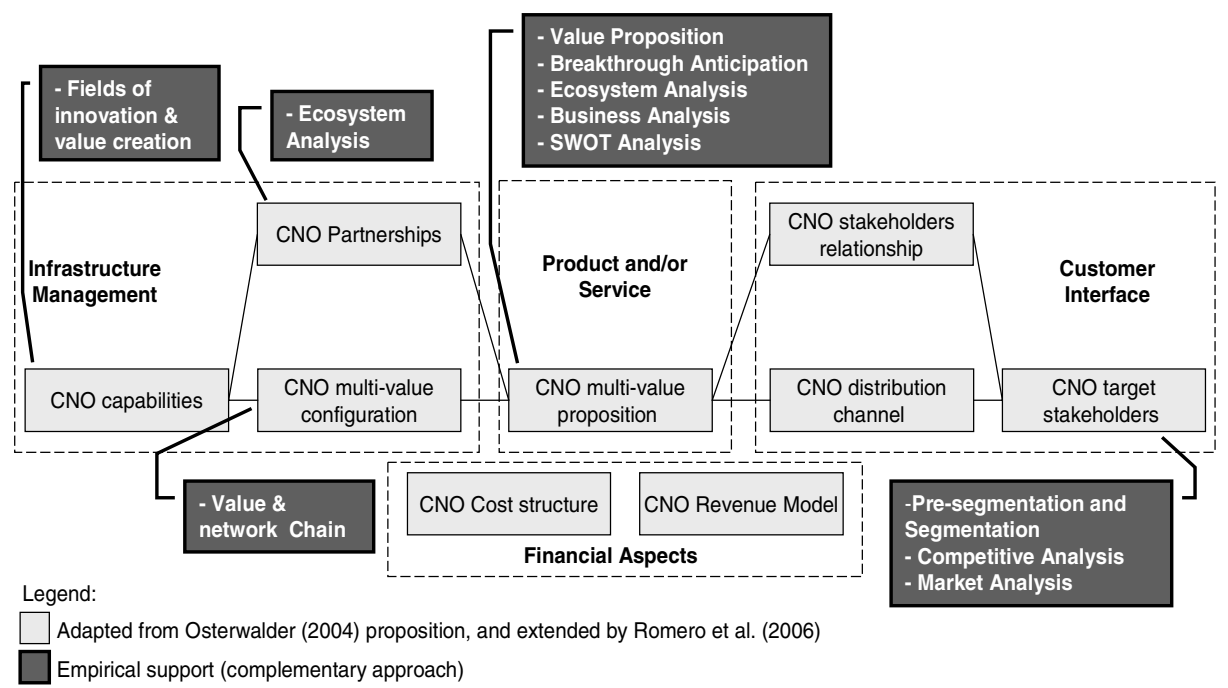

Fig. 2. Complementary tools and techniques applied to the approach proposed by Osterlwalder (2004) and extended to CNOs by Romero et al., (2006)

Nevertheless, the current approaches to develop BMs appear to be on a different wavelength from the current market landscape: especially when looking at new market dynamics caused by globalization, rising up of services in all activity sectors as well as open innovation processes. It is argued that new dynamics for BMs are required. As a result, next section introduces the concept of agile Business Models.

\section{Fostering Agile Business Models}

The motivation for this new perspective is spurred on the evolution from a value chain (Porter, 1985) where products definitions and customers' needs are stable and well defined ( $\mathrm{Li}$ et al., 2008) to a value network. The latter embraces any web of relationships that generates tangible and intangible value through complex dynamics between two or more individuals, groups or organizations (Allee, 2002).

As it was mentioned before, the current BMs used by CNOs operate in a rather linear "one-way flow". It implies that customers or end-users are not included in the dynamics of product/service life cycle management (from ideation to market). As a result, their involvement to the CNO's BM is to a certain extent passive. Table 1 
Table 1. Key features of Current BMs and Agile BMs

\begin{tabular}{l|l}
\hline \multicolumn{1}{c|}{ Current BMs (as-is) } & \multicolumn{1}{c}{ Agile BMs (to-be) } \\
\hline Static & Dynamic \\
\hline Financial aspects & Financial and social aspects \\
\hline Profit & $\begin{array}{l}\text { Profit / sustainability / effect of } \\
\text { commoditization }\end{array}$ \\
\hline Linear and Value chain & Value network / Digital ecosystems \\
\hline Value analysis and creation & Value creation and capture \\
\hline Product & $\begin{array}{l}\text { Increased development of services / Services } \\
\text { on top of the products }\end{array}$ \\
\hline Customer interfaces & Customer empowerment \\
\hline One to Many / One to one & Many to one \\
\hline
\end{tabular}

presents a holistic perspective about how conventional and/or current BMs in CNOs are traditionally focused (as-is) and how agile BMs are meant to be designed (to-be).

It is argued that an agile BM should be dynamic - to take into account the events that occur during and after its development. An agile BM should also consider the financial and social aspects that will impact the $\mathrm{CNO}$, so that it is possible to find out in which spheres of the social activities the CNO business might have influence. CNOs should also look for profit but without forgetting the sustainability requirements. Another relevant aspect is to be aware of the transformation of some goods and services into commodities: this shift in value has for sure an impact for CNOs in the sense that the competition will not be anymore on quality on these all of one kind products or services. This implies a search for additional differentiation features or a complete redefinition of the BM.

Indeed, the way of seeing the market (linear) needs to be reviewed. The evolution from a traditional value chain to a value network and digital ecosystems is essential: CNOs need to create an ecosystem promoting and enabling the sharing of ideas, knowledge and capabilities, as well as the evolution among services and solutions (Nachira et al., 2007). Another important aspect is that the development of a manufactured product is no longer enough. Services should be further developed and included on top of the products in order to create value to and with the customer. One example is the success of the Iphone which achieved a paramount breakthrough in the mobile landscape: it is of evidence that the device is stunningly beautiful which makes it attractive for all generations. But the genuine key success factor is the Apple Application Store with more than 25.000 applications and by March 2009, more than 500 million downloads. Service on top of the product is seen as a way to develop customer loyalty, i.e. in the Apple's case to lock-in customers on a single and unique application store which can not be overlooked and that no one can compete!

Finally, even if it is always of importance to capture and understand the customers' needs and requirements, this approach needs to be renewed. An interaction space is required where customers could participate through interactions with the CNO to product/service ideation or design. This can be achieved through changes suggestions, evaluation of new products/services. It implies to gather information and to analyze it, as well as to open communication channels to the customers. According to Prahalad et al. (2008), the behavior of individual customers and broad patterns of change must 
be understood. It has a direct impact on the infrastructure management, actors involved, financial aspects, quality of products and services.

Table 2 introduces what the authors have identified as levers and uncertainties for building agile business models in CNOs. The objective of this exercise is to find means to go beyond the concepts being used nowadays and to link them to a BM approach. These levers and uncertainties features are divided in three different categories: Organizational, Social, and Technological.

Table 2. Levers and uncertainties features for Agile Business Models

\begin{tabular}{|l|l|l|l|}
\cline { 2 - 4 } \multicolumn{1}{c|}{} & \multicolumn{1}{c|}{ Organizational } & \multicolumn{1}{c|}{ Social } & \multicolumn{1}{c|}{ Technological } \\
\hline Levers & $\begin{array}{l}\text { Business processes in } \\
\text { accordance to the } \\
\text { Business Models } \\
\text { Open Innovation } \\
\text { (crowdsourcing) }\end{array}$ & $\begin{array}{l}\text { Collective intelligence } \\
\text { User empowerment } \\
\text { (knowledge workers and } \\
\text { value co-creation) }\end{array}$ & $\begin{array}{l}\text { XaaS (SOA, web } \\
\text { services) Web X.0, P2P } \\
\text { Data analysis (analytics) }\end{array}$ \\
\hline $\begin{array}{l}\text { Uncer- } \\
\text { tainties }\end{array}$ & $\begin{array}{l}\text { Organizational culture } \\
\text { Quality and } \\
\text { maintenance }\end{array}$ & $\begin{array}{l}\text { Trust aspects } \\
\text { Intellectual Property } \\
\text { Rights } \\
\text { Sustainability }\end{array}$ & $\begin{array}{l}\text { Costs / time consuming } \\
\text { implementation } \\
\text { Return of Investments for } \\
\text { SMEs }\end{array}$ \\
\hline
\end{tabular}

On the Organizational side, it is argued that the (distributed) business processes should be developed in accordance with what is pointed out by the BM. It means that via the implementation of the strategies through their business processes, CNOs could be more agile and in a position to better react to new business opportunities. Another lever for an agile BM is the implementation of Open Innovation concept (Chesbrough, 2003), i.e. a subtle blend of internal and external innovation based on the subsequent acknowledgement: R\&D can seldom, if ever, be only internally developed implying that R\&D can be externally bought if this strategy brings advances to the BM.

However, impediments might occur due to the strength of the organizational culture. People inside the organization may not be used to this new open way of working: seeking the punctual help of an external expert for one specific opportunity can be interpreted as a threat by workers of the organization, or the process may not be modeled accurately causing bottlenecks to the CNO operation. Issues as quality and maintenance are also sensible to emergent approaches because they change the way the activities are performed.

Regarding the Social perspective, one may consider as a lever the power of collective intelligence in the Knowledge Economy (Brown and Hugh, 2001) as an important input which may modify the BM design. Individuals such as knowledge workers or active customers can provide content that may lead to changes in the BM. Collective intelligence rises up from the collaboration and competition, also called "coopetition" of many individuals, not necessarily being part of the CNO. CNOs may benefit from it for value creation and value co-creation (Prahalad et al., 2008).

Despite the importance of collective intelligence and user empowerment for value co-creation, issues like trust, intellectual property rights, and sustainability shall be carefully taken into consideration by CNOs. A clear definition of a value system model can provide support these issues. 
The latest perspective addressed in this table is the Technological one, which is seen as a horizontal building block that provides tools to support the organizational and social aspects of BMs. An agile BM shall then rely on a horizontal building block composed of an ICT base and of an analytic model. An analytic model aims to support innovation via the analysis of both structured and unstructured data in order to use and/or allocate resources according to the users' requirements (Prahalad et al., 2008). Analytics are then focused on the needs of single individuals $(\mathrm{N}=1)$ through global resources $(\mathrm{R}=\mathrm{G})$ and the decisions are based on information gathered from Web 2.0 expressions such as blogs, chat rooms, micro-blogs, and social networks in order to collect the customer's preferences and trends. Likewise, (distributed) business processes, supported by Service Oriented Architecture (SOA), can be swiftly reengineered and compliant with changes. The concept of XaaS ( $\underline{X} \underline{\text { as }} \underline{\text { a }} \underline{\text { Service}})$, where $X$ can be understood as "anything" also supports an agile BM. Application interaction in multi-levels among users, organizations and even machines bring a higher level of agility to CNOs. Web 2.0 tools can also support an agile business model by collecting information. It facilitates the communication among CNO partners and clients by providing collaboration tools and interoperability for information sharing.

The uncertainties related to the technological perspective involve the costs of providing this kind of structure, even when using already developed tools (Rabelo and Gusmeroli, 2008) and the time necessary in order to implement these solutions. Furthermore, it is not yet clear to SMEs how to measure the return of investments in technology.

Fig. 3 shows the interaction of the levers in the three dimensions aforementioned. It also considers the approach presented in Fig. 2. The approach presented in Fig. 3

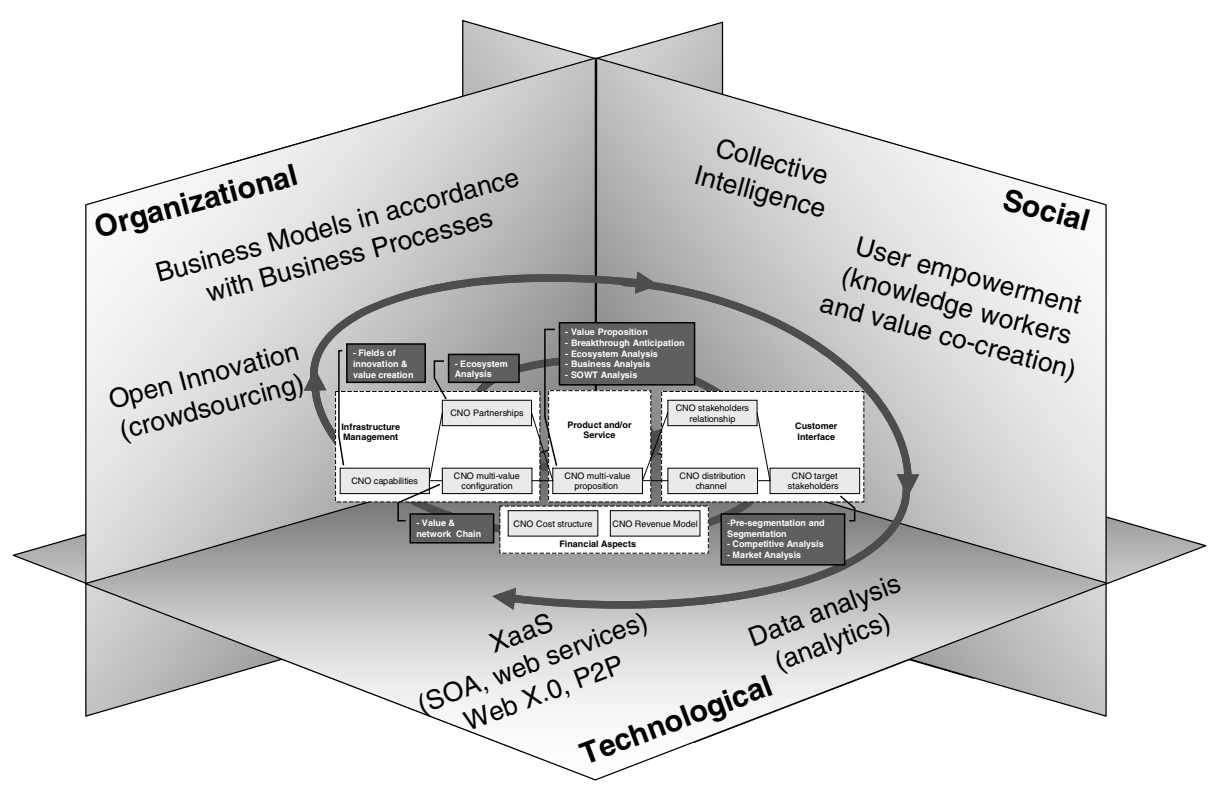

Fig. 3. Interaction of elements in Agile Business Models for CNOs 
intends to complement and update the existing ones. It aims at opening CNO's managers to the richness and changes provided by globalization and the Knowledge Economy. It also shows that the three dimensions are interrelated and require to be worked out in a holistic and integrated manner. For instance, Collective Intelligence (Social), Open Innovation (Organizational) and Analytics (Technological) need to be strongly coupled when these features are addressed. If one of these features is not integrated in the approach when defining a BM, a potential failure due to imbalance will occur. On the contrary, a harmonious combination of them will allow BMs to become more dynamic and will provide them agility to react to market uncertainties.

\section{Final Considerations}

This work brought to light the concept of an agile BM for CNOs. The paper started emphasizing how CNOs can be seen as an alternative in turbulent markets and periods of crisis. In the sequence the importance of BMs for the CNOs was presented. Reference to the conceptualization of BMs (model proposed by Chesbrough and Rosenboon, 2002) and the investigation of the evolution from a framework for BMs focused on one single enterprise (Osterwalder, 2004) to an attempt to a CNO BM (Romero et al., 2006) has been achieved to frame the scope and the advances proposed by the authors.

It was verified that despite the model presented by Romero et al., (2006) proposed a solid contribution to BMs in CNOs, it missed an agile perspective that may provide dynamicity for BMs in CNOs. Based on these observations, key features of current approaches for BMs and agile BMs were introduced. The levers and uncertainties that can affect an agile BM were also presented. These levers and uncertainties were split into three categories (Organizational, Social, and Technological) in order to cover a wider perspective.

With this agile business model approach, the authors aim to give insights and inspiration to further investigate the fundamentals of future BMs where complexity and adaptability will prevail. The next steps of this research work shall be dedicated to an exhaustive investigation of the levers and uncertainties with a field research to further articulate and illustrate in a mock-up organization, social, and technological dimensions.

\section{Acknowledgments}

The authors would like to thank Mrs. Sylvie Plantin, from Orange Labs R\&D, for her valuable comments.

\section{References}

1. Allee, V.: A Value Network Approach for Modelling and Measuring Intangibles. In: Proceedings Transparent Enterprise, Madrid (November 2002)

2. Brown, P., Hugh, L.: Capitalism and social progress: the future of society in a global economy. In: Brown, Lauder (eds.) Collective intelligence, Palgrave (2001)

3. Camarinha-Matos, L.M., Afsarmanesh, H.: Collaborative networks: a new scientific discipline. Journal of Intelligent Manufacturing 16, 439-452 (2005) 
4. Camarinha-Matos, L.M., Afsarmanesh, H.: Collaborative networks: value creation in a knowledge society. In: Wang, K., Kovács, G.L., Wozny, M.J., Fang, M. (eds.) PROLAMAT. IFIP, vol. 207, pp. 26-40. Springer, Heidelberg (2006)

5. Chesbrough, H., Rosenbloom, R.S.: The Role of the Business Model in Capturing Value from Innovation: Evidence from Xerox Corporation's Technology Spin-off Companies. Industrial and Corporate Change 11(3), 529-555 (2002)

6. Chesbrough, H.: Open Innovation: The New Imperative for Creating and Profiting from Technology. Harvard Business School Press, Boston (2003)

7. Lendrum, T.: The Strategic Partnering Handbook, A Practice Guide for Managers. McGraw-Hill, Nook Company, New York (1997)

8. Li, M., Crave, S., Grilo, A., van den Berg, R.: Unleashing the Potential of the European Knowledge Economy: Value Proposition for Enterprise Interoperability. European Commission - Informations Society and Media. Office for Official Publications of European Communities, Luxemburg (2008)

9. Loss, L., Pereira-Klen, A.A., Rabelo, R.J.: Value Creation Elements in Learning Collaborative Networked. In: Camarinha-Matos, L.M., Picard, W. (eds.) Pervasive Collaborative Networks. IFIP International Federation for Information Processing, vol. 283, pp. 75-84. Springer, Boston (2008)

10. Nachira, F., Dini, P., Nicolai, A., Le Louarn, M., Lèon, L.R.: Digital Business Ecosystems. Office for Official Publications of the European Communities, Luxembourg (2007) ISBN 92-79-01817-5

11. Oliver, C.: Determinants of inter-organizational relationships: integration and future direction. Academy of Management Review 15(2) (1990)

12. Osterwalder, A.: The Business Model Ontology - a proposition in a design science approach. PhD Thesis. Lausanne University, Switzerland (2004)

13. Papadakis, V.M., Kaloghirou, Y., Iatrelli, M.: Decision making from crisis to opportunity. Bus. Strategic Rev. 10(1), 29-37 (1999)

14. Plantin, et al: Orange Labs R\&D internal methodology for building business models (2008)

15. Porter, M.E.: Competitive advantage: creating and sustaining superior performance. Free Press, New York (1985)

16. Prahalad, C.K., Krishnan, M.S.: The New Age of Innovation: Driving Co-created Value Through Global Networks. McGraw-Hill Professional, New York (2008)

17. Rabelo, R.J., Gusmeroli, S.: The ECOLEAD Business Infrastructure for Netwirked Organizations. In: Camarinha-Matos, L.M., Picard, W. (eds.) Pervasive Collaborative Networks. IFIP International Federation for Information Processing, vol. 283, pp. 451-462. Springer, Boston (2008)

18. Romero, D., Galeano, N., Giraldo, J., Molina, A.: Towards the Definition of Business Models and Governance Rules for Virtual Breeding Environments. In: Camarinha-Matos, L.M., Afsarmanesh, H., Ollys, M. (eds.) Network-Centric Collaboration and Supporting Frameworks. International Federation for Information Processing (IFIP), vol. 224, pp. 103-110. Springer, New York (2006)

19. Stevenson, H., Jarillo, J.C.: A paradigm of entrepreneurship: Entrepreneurial management. Strategic Management J. 11, 17-27 (1990) 